\title{
GAIT CHARACTERISTICS OF ADULTS WITH INTELLECTUAL DISABILITIES
}

Alyt Oppewal PhD, Dederieke .A.M. Festen MD, PhD, Thessa I.M. Hilgenkamp PhD

Corresponding author:

Alyt Oppewal, PhD

E-mail address: a.oppewal@erasmusmc.nl

Erasmus MC, University Medical Center Rotterdam,

Department of General Practice, Intellectual Disability Medicine

P.O. Box 2040, 3000 CA Rotterdam, the Netherlands.

Dederieke A. Festen, MD, PhD

E-mail address: d.maes-festen@erasmusmc.nl

Erasmus MC, University Medical Center Rotterdam,

Department of General Practice, Intellectual Disability Medicine

P.O. Box 2040, 3000 CA Rotterdam, the Netherlands.

Thessa I.M. Hilgenkamp, PhD

E-mail address: t.hilgenkamp@erasmusmc.nl

Erasmus MC, University Medical Center Rotterdam,

Department of General Practice, Intellectual Disability Medicine

P.O. Box 2040, 3000 CA Rotterdam, the Netherlands.

\section{Acknowledgements}

The authors thank the management and professionals of the care organizations, Abrona (Huis ter Heide), Amarant (Tilburg) and Ipse de Bruggen (Zoetermeer), involved in the HA-ID consort for financing the study and their collaboration and support. We also thank the participants, their family members and caregivers for their collaboration. 


\begin{abstract}
Gait is a relevant and complex aspect of motor functioning. Disturbances are related to negative health outcomes. Gait characteristics of 31 adults with intellectual disabilities (ID) without Down syndrome (DS) (42.77 \pm 16.70 years) were investigated, and associations with age, sex, body mass index (BMI), and level of ID were assessed. Sex and BMI were significantly associated to some of the gait parameters, while age and level of ID were not. Gait characteristics of adults with ID seem to be comparable to those of the general older population who are on average 20 years older, except that adults with ID seem to spend less time in stance and double support phase and walk more variable and with a broader base of support. 120 words
\end{abstract}

\title{
Keywords
}

Intellectual disabilities, gait, adults 


\section{Introduction}

Gait is a highly relevant and complex aspect of motor functioning. It is not merely a simple motor activity, but it is an integrated cognitive and motor task (Hausdorff, Yogev, Springer, Simon, \& Giladi, 2005; Jahn, Zwergal, \& Schniepp, 2010) which relies on a constant interaction between the central and peripheral nervous system and the musculoskeletal system (Jahn et al., 2010). The gait pattern is the result of an optimization of propulsion, stability, shock absorption, and energy conservation (Perry, 1992), and disturbances in gait can lead to pain, instability, and an increased energy expenditure. Gait disturbances have been found to be common with advancing age and to predict future disability, falls, cognitive impairment, institutionalization, and mortality in the general population (Abellan van Kan et al., 2009; Verghese et al., 2006; Verghese, Wang, Lipton, Holtzer, \& Xue, 2007).

Because gait can be considered a complex cognitive task, and motor and cognitive functioning are fundamentally interrelated (Diamond, 2000), it is not surprising that gait disturbances are often seen in people with intellectual disabilities (ID) (Almuhtaseb, Oppewal, \& Hilgenkamp, 2014). An intellectual disability is characterized by significant limitations in both intellectual functioning (intelligence quotient [IQ] < 70) and adaptive behavior (American Association on Intellectual and Developmental Disabilities, 2016). In this population, motor development is also often delayed (Enkelaar, Smulders, van Schrojenstein Lantman-de Valk, Geurts, \& Weerdesteyn, 2012; Hartman, Houwen, Scherder, \& Visscher, 2010; Pereira, Basso, Lindquist, da Silva, \& Tudella, 2013; Rintala \& Loovis, 2013), and a lower IQ has been found to be associated with poorer motor performance (Smits-Engelsman \& Hill, 2012; Westendorp, Houwen, Hartman, \& Visscher, 2011).

Besides the cognitive impairment, people with ID also have a higher risk for gait disturbances due to other factors that are highly prevalent in this population. First, people with ID often have low physical activity (Hilgenkamp, Reis, van Wijck, \& Evenhuis, 2012; Peterson, Janz, \& Lowe, 2008; Temple, Frey, \& Stanish, 2006) and fitness levels (Hilgenkamp, van Wijck, \& Evenhuis, 2012; Oppewal, Hilgenkamp, van Wijck, \& Evenhuis, 2013). This can result in gait disorders because adequate fitness levels are needed for a proper gait. For example, cardiorespiratory fitness is important to keep walking for a certain amount of time (Rubino, 2002), and an adequate balance, strength, muscular endurance, and reaction time is necessary to keep an upright posture, bear bodyweight, and propel oneself forward while determining 
one's position in space (Cantor, 1999; Rubino, 2002). These are all necessary aspects for a proper gait. Second, obesity is highly prevalent in adults with ID (Bhaumik, Watson, Thorp, Tyrer, \& McGrother, 2008; de Winter, Bastiaanse, Hilgenkamp, Evenhuis, \& Echteld, 2012), which influences gait (Wearing, Hennig, Byrne, Steele, \& Hills, 2006). Obese adults show slower preferred walking speed and spend more time in stance and double support phase of the gait cycle which may help in maintaining balance (Wearing et al., 2006). Third, certain medications, such as antidepressants, antipsychotics, benzodiazepines, Parkinson medication, and antiarrhythmic drugs are known to negatively influence gait (Jahn et al., 2010; Rubino, 2002). The high medication use of people with ID, especially antipsychotics (de Kuijper et al., 2010) and frequent prevalence of polypharmacy (Evenhuis, 2014), may negatively influence their gait. Finally, neurological disturbances related to the etiology of the intellectual disability, such as spasticity and muscle hypotonia, can also result in impaired gait. This higher risk for gait impairments of people with ID may put them at increasing risk for the negative health outcomes mentioned previously. Therefore it is important to examine gait characteristics of people with ID to have a better understanding of the movement control and possible gait impairments seen in this population.

Most of the research regarding gait in people with ID has focused on people with Down syndrome (DS). These studies found that people with DS have lower values for gait cycle time, percent of swing in the gait cycle, step length, stride length, gait speed, and cadence than the general population, and higher values for step width, stride width, base of support, double support time, stance time, percent of stance and double support in the gait cycle, and show greater variability in their gait pattern than the general population (Almuhtaseb et al., 2014). These disturbances indicate that people with DS adapt their gait to compensate for a higher instability during walking, which can partly be explained by physical characteristics that accompany this genetic syndrome, such as ligament laxity and muscle hypotonia (Galli, Rigoldi, Brunner, Virji-Babul, \& Giorgio, 2008; Rigoldi et al., 2012; Rigoldi, Galli, Mainardi, Crivellini, \& Albertini, 2011).

These results from studies with people with DS are not generalizable to people with ID by other causes than DS, because of the specific physical characteristics related to this genetic syndrome. Only a few studies regarding gait in people with ID by other causes than DS have been performed. Chiba et al. (2009) and Haynes \& Lockhart (2012) found that adults with ID had lower values for gait speed and step 
length than the general population (Chiba et al., 2009; Haynes \& Lockhart, 2012). Salb et al. (2015) also found lower values for gait speed, step length and cadence in adults with ID compared to reference values of the general population (Salb et al., 2015). However, Sparrow et al. (1998) found higher values for gait speed and cadence, and lower values for stride length and duration in adults with ID in comparison to the general population. These studies give some indications of the gait characteristics of people with ID without DS, however results are not completely consistent across studies and only few gait parameters were assessed.

Therefore, the aim of this study was to investigate the gait characteristics of adults with ID from other causes than DS. Our secondary aim was to assess if the personal characteristics age, sex, BMI, and level of ID were associated with these gait characteristics, because in the general population an agerelated decline in gait is seen (Alexander, 1996; Verghese et al., 2006), sex differences are seen in certain gait characteristics (Bruening, Frimenko, Goodyear, Bowden, \& Fullenkamp, 2015; Hollman, McDade, \& Petersen, 2011), obesity is associated with altered gait characteristics (Wearing et al., 2006), and cognition is considered an important aspect of gait (Hausdorff et al., 2005; Jahn et al., 2010). We hypothesize that there is a great variability in the gait pattern of adults with ID and that gait parameters are associated with the personal characteristics age, sex, BMI, and level of ID. However, these associations may be less strong than in the general population because of possible other causes underlying the often lifelong gait abnormalities.

\section{Methods}

\section{Study design and participants}

This was a cross-sectional study executed in a consort of three ID care organizations in the Netherlands and the chair for Intellectual Disability Medicine of the Erasmus MC, University Medical Center Rotterdam. Participants were recruited from these three ID care organizations, resulting in 31 participants. All clients aged 20 years and over, with a mild $(I Q=50-69)$ or moderate $(I Q=35-49)$ ID, and the ability to walk without a walking aid were eligible to participate, except those with a diagnosis of DS, Parkinson's disease, cerebrovascular accident, dementia, Cerebral palsy, and a severe visual 
impairment (vision <0.3). Individuals with these diagnoses were excluded because we wanted to gain insight into the gait characteristics of adults with ID, without the influence of these specific neurological diagnoses. Adults with a severe visual impairment were excluded because of possible difficulties with and associated anxiety while performing the measurements. Behavioral therapists and medical doctors of the participating care organizations selected participants based on these inclusion and exclusion criteria. Data collection took place between December 2014 and July 2015.

The Medical Ethical Committee of the Erasmus MC, University Medical Center Rotterdam approved this study (MEC-2014-201). All participants or their legal representatives provided informed consent. This study was conducted according to the guidelines of the declaration of Helsinki (World Medical Association, 2013).

\section{Measurements}

\section{Personal characteristics}

Age, sex, and level of ID were retrieved from the behavioral therapists and medical doctors who selected the participants for the study. Level of ID was categorized as mild (IQ = 50 - 69) or moderate (IQ $=35-49)$ based on the International Classification of Diseases (ICD-10) criteria (WHO, 1996).

Height, weight, and leg length were measured during the data collection. Height was measured with a stadiometer with the participant wearing no shoes. Weight was measured with a digital floor scale with the participant wearing no shoes and light clothes. Body Mass Index (BMI) was calculated and categorized into normal $\left(<25 \mathrm{~kg} / \mathrm{m}^{2}\right)$, overweight $\left(25-30 \mathrm{~kg} / \mathrm{m}^{2}\right)$ and obese $\left(\geq 30 \mathrm{~kg} / \mathrm{m}^{2}\right)(\mathrm{WHO}, 1995)$. Leg length was measured from the greater trochanter to the floor, bisecting the lateral malleolus, with the participant wearing shoes.

To describe the study population in detail we also retrieved the following medical information and functional measures:

\section{Medical information}

The following medical information was retrieved from the medical files: presence of osteoarthritis, visual impairment, spasticity of the arms and/or legs, and the use of orthopedic shoes, antidepressants, 
antipsychotics, antiepileptics, benzodiazepines and polypharmacy (defined as using 5 or more medications).

\section{Functional assessment}

Short Physical Peformance Battery

The Short Physical Performance Battery is a strong predictor for institutionalization, disability, and mortality (Guralnik et al., 2000; Guralnik, Ferrucci, Simonsick, Salive, \& Wallace, 1995; Guralnik et al., 1994). The test battery is widely used in the general population and consists of a measure of gait speed, three balance stances (side by side, semi-tandem stance, tandem stance), and the 5 times chair stand. A total score is calculated for these tests with a range from 0 to 12 points, with 12 points being the best performance.

Falls

Falls were registered for three months using monthly fall registration calendars. Participants put a sticker on their calendar each day; a green sticker if they did not experience a fall that day, and a red sticker if they did experienced a fall. If the participant was not able to do this himself, they were assisted by their professional caregiver. At the end of the week, their professional caregivers checked the calendars and checked of the week with a smiley face sticker. We collected the fall calendars every month.

\section{Gait measurements}

Gait was assessed with the GAITRite Electronic Walkway (CIR Systems, Inc., USA; 5.79m with $4.88 \mathrm{~m}$ active area, $120 \mathrm{~Hz}$ scan rate), a roll-up carpet with pressure sensor pads that are activated by the pressure of footfalls. The GAITRite is able to reliably and validly measure temporal and spatial gait parameters (Bilney, Morris, \& Webster, 2003; Kressig, Beauchet, \& European GAITRite Network Group, 2006; Menz, Latt, Tiedemann, Mun San Kwan, \& Lord, 2004; van Uden \& Besser, 2004). Test-retest reliability has also been established in people with DS (Gretz et al., 1998) and elderly with a mild cognitive impairment (Montero-Odasso et al., 2009). The parameters that were measured are described in table 1. The parameters included mean spatial and temporal parameters over multiple steps, and the variability over these steps measured as the standard deviations. 
[Table 1]

\section{Procedure}

Data were collected at locations close and/or familiar to the participants; in a large room or a gym at the care organizations. To assure safety during testing, the Revised Physical Activity Readiness Questionnaire (rPAR-Q) was administered prior to participation (Cardinal, Esters, \& Cardinal, 1996; Thomas, Reading, \& Shephard, 1992). Professional caregivers answered the questions, if any of the questions were answered with 'yes' or 'unknown', the medical physician was contacted to determine whether the participant could safely perform the measurements.

Measurements were performed by a human movement scientist and physiotherapists with experience with people with ID. All gait measurements were performed by the same test instructor.

The GAITRite was placed in the test location with two meter in front of and at the end of it to avoid acceleration and deceleration on the GAITRite, according to the guidelines (Kressig et al., 2006). Participants were instructed to walk at their own comfortable walking speed. Four walks were performed, of which the first walk was considered a practice walk. Participants walked with shoes. After the gait measurements, the height, weight, leg length and functional measurements were performed.

\section{Statistical analyses}

Personal characteristics, medical information and gait parameters were described for the total group. For analyses of the gait parameters, the practice walk was excluded and the remaining three walks were used. The mean of both legs across the three walks were taken for all the parameters.

For further analyses normality of the gait parameters was checked and considered sufficient. To assess if the personal characteristics were related to the gait parameters, all gait parameters were adjusted for leg length by dividing the gait parameters by the mean leg length of the participants. Independent $t$ tests were used to assess differences in gait parameters between females and males and between adults with mild and with moderate ID. Effect sizes were calculated for each comparison with Cohen's $d$ (Cohen, 1992). Effect size values of 0.2, 0.5 , and 0.8 were considered as benchmarks for 
small, medium, and large effect sizes, respectively. The influence of age and BMI on the gait parameters were assessed with the Pearson's correlation coefficient $(r)$, which also is a measure of the size of effect. $R$ values of $0.1,0.3$, and 0.5 were considered as benchmarks for small, medium, and large effect sizes, respectively (Cohen, 1992). Bonferroni correction was used to correct for multiple testing. $P$-values smaller than $0.002(0.05 / 27$ gait parameters) were considered statistically significant. Because of the known association between sex and BMI, multiple linear regression analyses were performed for those gait parameters associated with both sex and BMI $(p<0.05)$ to assess the independent association of sex and BMI with these gait parameters.

Analyses were performed with the Statistical Package for Social Sciences (SPSS) version 21 (IBM Corporation, New York).

\section{Results}

\section{Personal characteristics and medical information}

The personal characteristics and medical information of the study sample are described in table 2. The mean age of the sample was $42.77 \pm 16.7$ (range $20-68$ ), and $77.4 \%$ was male. Almost half of the study sample had a mild ID (48.4\%).

[Table 2]

\section{Gait parameters}

After combining the three walks while walking at comfortable speed, an average of 18.71 steps \pm 4.46 was available per participant, with a range of 12 to 33 steps. The mean gait parameters of these walks are presented in table 3.

\section{Associations with sex}

Females had a significantly higher standard deviation of step time than males (females $0.03 \pm$ 0.01 , range $0.03-0.04$ vs males $0.02 \pm 0.01$, range $0.01-0.04 ; t(29)=-3.55, p=0.001, d=2.04)$. The 
other gait parameters did not differ significantly between females and males, after correction for multiple testing $(p<0.002)$. However, when looking at the large effect sizes (table 4$)$ we see that females had a higher double support time (females $0.23 \pm 0.06$, range $0.13-0.33$ vs males $0.20 \pm 0.06$, range $0.07-$ $0.35 ; t(29)=-1.80, p=0.082, d=0.81$ ), and stance time (females $59.81 \pm 2.07$, range $56.05-62.05$ vs males $58.73 \pm 1.94$, range $53.60-62.90 ; t(29)=-3.25, p=0.003, d=1.40)$ and double support time (females $20.08 \pm 4.27$, range $12.0-24.70$ vs males $17.49 \pm 3.92$, range $6.70-26.20 ; t(29)=-2.27, p=$ $0.031, d=0.98$ ) as a percentage of the gait cycle time, and had higher standard deviations of stride time (females $0.05 \pm 0.01$, range $0.03-0.07$ vs males $0.03 \pm 0.01$, range $0.01-0.06 ; t(29)=-2.65, p=0.013$, $d=1.18$ ), swing time (females $0.03 \pm 0.01$, range $0.02-0.04$ vs males $0.02 \pm 0.01$, range $0.01-0.05$; $t(29)=-2.47, p=0.019, d=1.04$ ) and single support time (females $0.03 \pm 0.01$, range $0.02-0.04$ vs males $0.02 \pm 0.01$, range $0.01-0.05 ; t(29)=-2.47, p=0.019, d=1.04)$ than males. Medium effect sizes were found for step length, stride length, cadence, step time, stride (cycle) time, stance time, single support time, single support as a percentage of the gait cycle time and standard deviation of double support time.

\section{Associations with level of ID}

No significant differences were found in gait parameters between adults with mild ID and moderate ID, and a medium effect size was only seen for step length and the standard deviation for base of support. The rest of the effect sizes were small (table 4).

\section{Associations with age}

None of the correlations between age and the gait parameters were significant, and all of the effect sizes were small (table 4).

\section{Associations with BMI}

A higher BMI was significantly correlated with higher double support time $(r=0.67, p<0.001)$ and higher double support time as a percentage of the gait cycle time $(r=0.730, p<0.001)$. A medium positive effect size was seen for the associations between BMI and stance time, stance as a percentage of the gait cycle time, and standard deviation of double support time. 
Multiple linear regression analyses

Both sex and BMI were associated with stance $(p<0.05)$ and double support time $(\operatorname{sex} p<0.05$; BMI $p<0.002)$ as a percentage of the gait cycle time. The multiple regression analyses with both sex and $\mathrm{BMI}$ in the model showed that BMI did not remain significantly associated with stance time as a percentage of the gait cycle time, and sex did not remain significantly associated with double support time as a percentage of the gait cycle time.

[Table 3]

[Table 4]

\section{Discussion}

This study describes a large number of gait characteristics for adults with intellectual disabilities (ID) by other causes than Down syndrome (DS). In addition, we assessed if the personal characteristics age, sex, BMI, and level of ID were associated with the gait characteristics. The only associations that reached significance were those between sex and the standard deviation of step time, which was higher for females, and a higher BMI with higher double support time and higher double support time as a percentage of the gait cycle time.

When comparing the gait characteristics found in this study to the few (step length, stride length, velocity and cadence) studied in previous studies regarding adults with ID without DS, we see that step length (Chiba et al., 2009; Haynes \& Lockhart, 2012), stride length (Salb et al., 2015), velocity (Chiba et al., 2009; Haynes \& Lockhart, 2012; Salb et al., 2015), and cadence (Salb et al., 2015) of our sample was higher than in these other studies. The study samples of these studies differed from ours, which may explain some of the differences. The studies of Haynes \& Lockhart (2012) and Chiba et al. (2009) included mostly adults with severe and profound ID, and the study sample of Salb et al. (2015) was on average older (59.59 SD 16.71 years) than our sample. However, because these studies only measured a few gait variables and we were therefore only able to compare only these few gait variables, and study samples were rather small, it is too early to draw firm conclusions.

Most of the studies on gait in people with ID studied people with DS. Comparing our results to 
those found in adults with DS, with a comparable mean age of the study sample, we found a higher stride length (Smith, Ashton-Miller, \& Ulrich, 2010; Smith \& Ulrich, 2008), velocity (Smith \& Ulrich, 2008), step time (Gretz et al., 1998), stride (cycle) time (Gretz et al., 1998), and single support time as a percentage of the gait cycle (Gretz et al., 1998), and smaller base of support (Gretz et al., 1998; Smith et al., 2010; Smith \& Ulrich, 2008), and lower stance time (Smith et al., 2010; Smith \& Ulrich, 2008) and double support time (Gretz et al., 1998; Smith \& Ulrich, 2008) as a percentage of the gait cycle. Overall, it seems that adults with ID walk with a higher velocity and take bigger strides in less time, with a smaller base of support and less time spent in the stance and double support phase during the gait cycle than adults with DS, which represents a better gait pattern.

Looking at other genetic syndromes, two studies looked at gait in people with Prader-Willi syndrome (Cimolin et al., 2010; Vismara et al., 2007). In comparison to these studies, we found a higher velocity and single support time as a percentage of the gait cycle, and a lower cadence and stance time as a percentage of the gait cycle. Two other studies looked at gait in people with Williams syndrome (Hocking, McGinley, Moss, Bradshaw, \& Rinehart, 2010; Hocking, Rinehart, McGinley, \& Bradshaw, 2009). In comparison to these studies we found a higher stride length, and lower cadence, and double support time as a percentage of the gait cycle. Our base of support and velocity was somewhat lower or comparable to the values found in these studies.

In a large study in the general population including 1500 community-dwelling adults with a mean age of $68.8 \pm 10.1$ the same gait parameters were measured as we did, except for toe in/ toe out and stride velocity (Verlinden et al., 2013). Comparing our results to this study, the gait parameters of adults with ID looked rather similar to those of this general older population, except for the following variables. Adults with ID showed a higher base of support, swing time, single support time, swing time and single support time as a percentage of the gait cycle, and standard deviations of stride length and stride velocity, and lower double support time, and stance time and double support time as a percentage of the gait cycle. The gait characteristics of adults with ID seem to be rather similar to those of the general older population who are on average 20 years older, except that adults with ID seem to spend less time in the stance and double support phase of the gait cycle and walk more variable with a broader base of support.

The higher standard deviations observed in our study, when comparing these to those seen in the 
general older population, represent a higher variability in the gait pattern of adults with ID. This higher variability has also been found in studies regarding people with DS (Black, Smith, Wu, \& Ulrich, 2007; Buzzi \& Ulrich, 2004; Smith, Stergiou, \& Ulrich, 2011). In healthy adults, stride-to-stride variability is relatively small. Impairments in the gait regulating systems may lead to increased stride-to-stride variability, for example with neurodegenerative diseases such as Parkinson's and Alzheimer's disease (Hausdorff, 2005). Gait variability measures have an important clinical aspect, because they predict future falls and mobility related disability (Brach, Studenski, Perera, VanSwearingen, \& Newman, 2007; Hausdorff, 2005; Hausdorff, Rios, \& Edelberg, 2001). However, the higher gait variability of people with ID may also be beneficial. Studies regarding people with DS, suggested that this higher variability may represent a compensation strategy to compensate for their limitations (such as joint laxity and hypotonia) by using this variability to adapt their gait as optimal as possible during walking (Black et al., 2007; Smith et al., 2011). Future studies should explore the clinical consequences of this higher gait variability in people with ID.

In the general population, an age-related decline is seen in gait (Alexander, 1996; Verghese et al., 2006). However, age was not associated to gait characteristics in our study. This may be because our study sample might be too young to find a real association with age, because in the general population a decline in gait becomes more evident after the age of 60 years (Alexander, 1996; Jahn et al., 2010). It may also be that gait characteristics of people with ID are more related to their lifelong cognitive impairment which may influence their motor development since childhood. Resulting in gait abnormalities at a younger age, which may therefore be less dependent on an age-related decline. This is supported by the finding that the gait characteristics of our study sample looked already rather similar to those of older adults in the general older population of 20 years older.

However, the level of ID was not associated to the gait characteristics, although a medium effect size was found for step length and the standard deviation of base of support. Cognition is considered an important aspect of gait, and functional imaging studies have revealed the subcortical and cortical areas that are involved in the control of gait (Hausdorff et al., 2005; Jahn et al., 2010; Rosano et al., 2008). Especially executive functions have been found to be related to gait impairments (Alexander \& Hausdorff, 2008). The categories mild and moderate ID (based on IQ) may therefore have been too broad to find an 
association with the gait parameters. Walking while dual tasking is often used to assess the influence of cognition on gait, and this may be a more sensitive way to assess the effect of cognition on gait in adults with ID. Our study group will address this in a following study.

Sex was associated with some gait characteristics. Looking at the large effect sizes, females spent more time in the stance and double support phase of the gait cycle and had higher standard deviations, representing more variability in their gait parameters. However, only the difference in standard deviation of step time remained significant after correction for multiple testing. When controlling for BMI, the association between sex and stance time as a percentage of the gait cycle time still remained $<0.05$, but the association with double support time as a percentage of the gait cycle time did not. Even though most of the associations did not remain significant after correcting for multiple testing, finding large effect sizes regarding the differences between females and males with only 7 females in the study is remarkable. But with only 7 females it remains difficult to generalize these findings and draw firm conclusions.

A higher BMI was significantly associated with higher double support time, both absolute and as a percentage of the gait cycle time. This was also seen in a medium effect size for a higher stance time (both absolute and as a percentage of the gait cycle time) with higher BMI (although non-significant after correcting for multiple testing). When correcting for sex, BMI was still significantly associated with double support time as a percentage of the gait cycle time. These results are comparable to those found in the general population, where adults with obesity showed to spent more time in the stance and double support phase of the gait cycle (Wearing et al., 2006). In the general population obesity was also found to result in a slower walking speed, reduced step length and step frequency, and a greater step width (Wearing et al., 2006), which was not found in our study. These changes in gait with obesity are thought to help in maintaining balance (Wearing et al., 2006). Implications of impaired gait can be a higher energetic cost. Impaired gait has been found to be related to higher energy expenditure during walking in people with ID (Ohwada, Nakayama, Suzuki, Yokoyama, \& Ishimaru, 2005) (Lante, Reece, \& Walkley, 2010) and DS (Agiovlasitis, McCubbin, Yun, Pavol, \& Widrick, 2009; Agiovlasitis et al., 2011). This higher energetic cost may be the result of excessive body movements and disturbed gait kinematics (Agiovlasitis, McCubbin, Yun, Widrick, \& Pavol, 2015; Ohwada et al., 2005). Agiovlasitis et al. (2015) 
found that differences in gait characteristics between adults with DS and adults of the general population accounted for $73.9 \%$ of the variance in net metabolic rate between the groups. Because the gait characteristics of our sample seem rather comparable to those found in a population of the general population aged 20 years older, and an increased energy cost during walking is seen with age-related gait changes (VanSwearingen \& Studenski, 2014), this may suggest a higher energy cost of walking in adults with ID. This higher energy expenditure may cause people with ID to avoid activities, and thereby may contribute to the low physical activity levels seen in this population (Hilgenkamp, Reis, et al., 2012; Peterson et al., 2008; Temple et al., 2006), which in turn negatively influences their health. This has not yet been investigated in people with ID, but in adults with cerebral palsy it was found that people with high physical strain during walking at their preferred walking speed were likely to walk less in their daily life (Slaman et al., 2013). If this also holds true for people with ID, this is an important aspect to take into account when developing and implementing interventions to increase physical activity in this population.

Because gait characteristics influence the energetic cost of walking, and gait can be considered a marker of global health with disturbances in gait predicting future disability, falls, cognitive impairment, institutionalization, and mortality in the general population (Abellan van Kan et al., 2009; cLord et al., 2013; Verghese et al., 2006; Verghese et al., 2007), it is important to investigate how gait can be improved in people with ID. It has been found that people with ID have low physical activity and physical fitness levels across the lifespan (Golubovic, Maksimovic, Golubovic, \& Glumbic, 2012; Hilgenkamp, Reis, et al., 2012; Hilgenkamp, van Wijck, et al., 2012; Lahtinen, Rintala, \& Malin, 2007; Oppewal et al., 2013; Salaun \& Berthouze-Aranda, 2012; Temple et al., 2006) which could influence their gait. Being inactive and less fit from a young age, along with a cognitive impairment, limits the opportunities to develop motor skills, which can be seen in less developed locomotor skills and a later onset of walking in people with ID (Enkelaar et al., 2012; Hartman et al., 2010; Rintala \& Loovis, 2013). Creating a learning environment to stimulate motor development may be beneficial for improving gait of people with ID. Physical fitness and exercise have been found to be related to multiple health and functional components (Bartlo \& Klein, 2011; Oppewal, Hilgenkamp, van Wijck, Schoufour, \& Evenhuis, 2014, 2015; Ringenbach et al., 2016; van Schijndel-Speet, Evenhuis, van Wijck, van Montfort, \& Echteld, 2016), and exercise programs may also be effective in improving gait in people with ID. However, this has barely been studied in this 
population. One recent study found that an 8-week balance exercise program (twice a week, 40-min sessions) was effective in improving gait characteristics with an increase in velocity, step length, stride length, and a decrease cadence, step time and stride time children with mild ID (15.2 SD 2.2 years) (Lee, Lee, Shin, Shin, \& Song, 2014). Treadmill training has also been found to improve gait in people with DS, and to produce a faster onset of walking and an improved walking pattern in infants with DS (Enkelaar et al., 2012). Additional research regarding interventions to improve gait in adults with ID is needed. In addition, research regarding the association between physical fitness and gait characteristics is needed to identify which physical fitness components could be most important to train with regard to improving gait.

This study was one of the first studies to assess gait characteristics in adults with ID by other causes than DS. A strong aspect of this study is the large amount of gait variables investigated. However, this study had some limitations. Due to the small sample size, heterogeneous sample, and the limited number of females, the results may not be representative for the targeted population of adults with ID. In addition, this study sample only included participants that received care from a care organization and lived in the central settings of these organizations. By providing important participant characteristics that may influence gait, we tried to facilitate the interpretation of these results and comparison against future studies. The small sample size may have also resulted in a low statistical power to find significant associations. Therefore, effect sizes were also presented to give insight in the magnitude of the effect of age, sex, BMI, and level of ID on the gait characteristics.

In conclusion, this study provided an overview of gait characteristics of adults with ID from other causes than DS. Some sex differences and significant associations with BMI were seen, but age and level of ID were not associated with gait. To understand the impact of the gait characteristics of adults with ID future studies should focus on the relation between gait characteristics and negative health outcomes in adults with ID, such as higher energy expenditure, falls, and future disability.

\section{Conflict of interest}

None. 


\section{References}

Abellan van Kan, G., Rolland, Y., Andrieu, S., Bauer, J., Beauchet, O., Bonnefoy, M., . . Vellas, B. (2009). Gait speed at usual pace as a predictor of adverse outcomes in community-dwelling older people an International Academy on Nutrition and Aging (IANA) Task Force. J Nutr Health Aging, 13(10), 881-889.

Agiovlasitis, S., McCubbin, J. A., Yun, J., Pavol, M. J., \& Widrick, J. J. (2009). Economy and preferred speed of walking in adults with and without Down syndrome. Adapt Phys Activ Q, 26(2), 118-130.

Agiovlasitis, S., McCubbin, J. A., Yun, J., Widrick, J. J., \& Pavol, M. J. (2015). Gait characteristics of adults with Down syndrome explain their greater metabolic rate during walking. Gait Posture, 41(1), 180-184.

Agiovlasitis, S., Motl, R. W., Ranadive, S. M., Fahs, C. A., Yan, H., Echols, G. H., . . Fernhall, B. (2011). Energetic optimization during over-ground walking in people with and without Down syndrome. Gait Posture, 33(4), 630-634.

Alexander, N. B. (1996). Gait disorders in older adults. J Am Geriatr Soc, 44(4), 434-451.

Alexander, N. B., \& Hausdorff, J. M. (2008). Guest editorial: linking thinking, walking, and falling. J Gerontol A Biol Sci Med Sci, 63(12), 1325-1328.

Almuhtaseb, S., Oppewal, A., \& Hilgenkamp, T. I. M. (2014). Gait characteristics in individuals with intellectual disabilities: A literature review. Res Dev Disabil, 35(11), 2858-2883. doi:http://dx.doi.org/10.1016/j.ridd.2014.07.017

American Association on Intellectual and Developmental Disabilities. (2016). Definiton of Intellectual Disability. Retrieved from http://www.aamr.org/

Bartlo, P., \& Klein, P. J. (2011). Physical activity benefits and needs in adults with intellectual disabilities: systematic review of the literature. Am J Intellect Dev Disabil, 116(3), 220-232.

Bhaumik, S., Watson, J. M., Thorp, C. F., Tyrer, F., \& McGrother, C. W. (2008). Body mass index in adults with intellectual disability: distribution, associations and service implications: a populationbased prevalence study. J Intellect Disabil Res, 52(Pt 4), 287-298.

Bilney, B., Morris, M., \& Webster, K. (2003). Concurrent related validity of the GAITRite ${ }^{\circledR}$ walkway system for quantification of the spatial and temporal parameters of gait. Gait \& Posture, 17(1), 68-74. doi:http://dx.doi.org/10.1016/S0966-6362(02)00053-X

Black, D. P., Smith, B. A., Wu, J., \& Ulrich, B. D. (2007). Uncontrolled manifold analysis of segmental angle variability during walking: preadolescents with and without Down syndrome. Exp Brain Res, 183(4), 511-521.

Brach, J. S., Studenski, S. A., Perera, S., VanSwearingen, J. M., \& Newman, A. B. (2007). Gait variability and the risk of incident mobility disability in community-dwelling older adults. J Gerontol A Biol Sci Med Sci, 62(9), 983-988.

Bruening, D. A., Frimenko, R. E., Goodyear, C. D., Bowden, D. R., \& Fullenkamp, A. M. (2015). Sex differences in whole body gait kinematics at preferred speeds. Gait \& Posture, 41(2), 540-545. doi:http://dx.doi.org/10.1016/i.gaitpost.2014.12.011

Buzzi, U. H., \& Ulrich, B. D. (2004). Dynamic stability of gait cycles as a function of speed and system constraints. Motor Control, 8(3), 241-254.

Cantor, C. R. (1999). Gait disorders. Clin Podiatr Med Surg, 16(1), 141-151.

Cardinal, B. J., Esters, J., \& Cardinal, M. K. (1996). Evaluation of the revised physical activity readiness questionnaire in older adults. Med Sci Sports Exerc, 28(4), 468-472.

Chiba, Y., Shimada, A., Yoshida, F., Keino, H., Hasegawa, M., Ikari, H., . . Hosokawa, M. (2009). Risk of fall for individuals with intellectual disability. Am J Intellect Dev Disabil, 114(4), 225-236.

Cimolin, V., Galli, M., Grugni, G., Vismara, L., Albertini, G., Rigoldi, C., \& Capodaglio, P. (2010). Gait patterns in Prader-Willi and Down syndrome patients. J Neuroeng Rehabil, 7, 28.

cLord, S., Galna, B., Verghese, J., Coleman, S., Burn, D., \& Rochester, L. (2013). Independent domains of gait in older adults and associated motor and nonmotor attributes: validation of a factor analysis approach. J Gerontol A Biol Sci Med Sci, 68(7), 820-827.

Cohen, J. (1992). A power primer. Psychol Bull, 112(1), 155-159.

de Kuijper, G., Hoekstra, P., Visser, F., Scholte, F. A., Penning, C., \& Evenhuis, H. (2010). Use of antipsychotic drugs in individuals with intellectual disability (ID) in the Netherlands: prevalence and reasons for prescription. J Intellect Disabil Res, 54(7), 659-667. 
de Winter, C. F., Bastiaanse, L. P., Hilgenkamp, T. I., Evenhuis, H. M., \& Echteld, M. A. (2012). Overweight and obesity in older people with intellectual disability. Res Dev Disabil, 33(2), 398405.

Diamond, A. (2000). Close interrelation of motor development and cognitive development and of the cerebellum and prefrontal cortex. Child Dev, 71(1), 44-56.

Enkelaar, L., Smulders, E., van Schrojenstein Lantman-de Valk, H., Geurts, A. C., \& Weerdesteyn, V. (2012). A review of balance and gait capacities in relation to falls in persons with intellectual disability. Res Dev Disabil, 33(1), 291-306.

Evenhuis, H. M. (2014). [Poor health at an earlier age: frailty in people with intellectual disabilities]

Niet eerder oud, maar eerder ongezond: de kwetsbaarheid van mensen met verstandelijke beperkingen. Ned Tijdschr Geneeskd, 158, A8016.

Galli, M., Rigoldi, C., Brunner, R., Virji-Babul, N., \& Giorgio, A. (2008). Joint stiffness and gait pattern evaluation in children with Down syndrome. Gait Posture, 28(3), 502-506.

Golubovic, S., Maksimovic, J., Golubovic, B., \& Glumbic, N. (2012). Effects of exercise on physical fitness in children with intellectual disability. Res Dev Disabil, 33(2), 608-614.

Gretz, H. R., Doering, L. L., Quinn, J., Raftopoulos, M., Nelson, A. J., \& Zwick, D. E. (1998). Functional ambulation performance testing of adults with Down syndrome. NeuroRehabilitation, 11(3), 211225.

Guralnik, J. M., Ferrucci, L., Pieper, C. F., Leveille, S. G., Markides, K. S., Ostir, G. V., . . Wallace, R. B. (2000). Lower extremity function and subsequent disability: consistency across studies, predictive models, and value of gait speed alone compared with the short physical performance battery. $\mathrm{J}$ Gerontol A Biol Sci Med Sci, 55(4), M221-231.

Guralnik, J. M., Ferrucci, L., Simonsick, E. M., Salive, M. E., \& Wallace, R. B. (1995). Lower-extremity function in persons over the age of 70 years as a predictor of subsequent disability. $N$ Engl $\mathrm{J}$ Med, 332(9), 556-561.

Guralnik, J. M., Simonsick, E. M., Ferrucci, L., Glynn, R. J., Berkman, L. F., Blazer, D. G., . . Wallace, R. B. (1994). A short physical performance battery assessing lower extremity function: association with self-reported disability and prediction of mortality and nursing home admission. $J$ Gerontol, 49(2), M85-94.

Hartman, E., Houwen, S., Scherder, E., \& Visscher, C. (2010). On the relationship between motor performance and executive functioning in children with intellectual disabilities. J Intellect Disabil Res, 54(5), 468-477.

Hausdorff, J. M. (2005). Gait variability: methods, modeling and meaning. J Neuroeng Rehabil, 2, 19.

Hausdorff, J. M., Rios, D. A., \& Edelberg, H. K. (2001). Gait variability and fall risk in community-living older adults: a 1-year prospective study. Arch Phys Med Rehabil, 82(8), 1050-1056.

Hausdorff, J. M., Yogev, G., Springer, S., Simon, E. S., \& Giladi, N. (2005). Walking is more like catching than tapping: gait in the elderly as a complex cognitive task. Exp Brain Res, 164(4), 541-548.

Haynes, C. A., \& Lockhart, T. E. (2012). Evaluation of gait and slip parameters for adults with intellectual disability. J Biomech, 45(14), 2337-2341.

Hilgenkamp, T. I., Reis, D., van Wijck, R., \& Evenhuis, H. M. (2012). Physical activity levels in older adults with intellectual disabilities are extremely low. Res Dev Disabil, 33(2), 477-483.

Hilgenkamp, T. I., van Wijck, R., \& Evenhuis, H. M. (2012). Low physical fitness levels in older adults with ID: Results of the HA-ID study. Res Dev Disabil, 33(4), 1048-1058.

Hocking, D. R., McGinley, J. L., Moss, S. A., Bradshaw, J. L., \& Rinehart, N. J. (2010). Effects of external and internal cues on gait function in Williams syndrome. J Neurol Sci, 291(1-2), 57-63.

Hocking, D. R., Rinehart, N. J., McGinley, J. L., \& Bradshaw, J. L. (2009). Gait function in adults with Williams syndrome. Exp Brain Res, 192(4), 695-702.

Hollman, J. H., McDade, E. M., \& Petersen, R. C. (2011). Normative spatiotemporal gait parameters in older adults. Gait \& Posture, 34(1), 111-118. doi:http://dx.doi.org/10.1016/j.gaitpost.2011.03.024

Jahn, K., Zwergal, A., \& Schniepp, R. (2010). Gait disturbances in old age: classification, diagnosis, and treatment from a neurological perspective. Dtsch Arztebl Int, 107(17), 306-315; quiz 316.

Kressig, R. W., Beauchet, O., \& European GAITRite Network Group. (2006). Guidelines for clinical applications of spatio-temporal gait analysis in older adults. Aging Clin Exp Res, 18(2), 174-176.

Lahtinen, U., Rintala, P., \& Malin, A. (2007). Physical performance of individuals with intellectual disability: a 30 year follow up. Adapt Phys Activ Q, 24(2), 125-143. 
Lante, K., Reece, J., \& Walkley, J. (2010). Energy expended by adults with and without intellectual disabilities during activities of daily living. Res Dev Disabil, 31(6), 1380-1389.

Lee, K. J., Lee, M. M., Shin, D. C., Shin, S. H., \& Song, C. H. (2014). The effects of a balance exercise program for enhancement of gait function on temporal and spatial gait parameters in young people with intellectual disabilities. J Phys Ther Sci, 26(4), 513-516.

Menz, H. B., Latt, M. D., Tiedemann, A., Mun San Kwan, M., \& Lord, S. R. (2004). Reliability of the GAITRite walkway system for the quantification of temporo-spatial parameters of gait in young and older people. Gait Posture, 20(1), 20-25.

Montero-Odasso, M., Casas, A., Hansen, K. T., Bilski, P., Gutmanis, I., Wells, J. L., \& Borrie, M. J. (2009). Quantitative gait analysis under dual-task in older people with mild cognitive impairment: a reliability study. J Neuroeng Rehabil, 6, 35.

Ohwada, H., Nakayama, T., Suzuki, Y., Yokoyama, T., \& Ishimaru, M. (2005). Energy expenditure in males with mental retardation. J Nutr Sci Vitaminol (Tokyo), 51(2), 68-74.

Oppewal, A., Hilgenkamp, T. I., van Wijck, R., \& Evenhuis, H. M. (2013). Cardiorespiratory fitness in individuals with intellectual disabilities—A review. Res Dev Disabil, 34(10), 3301-3316. doi:http://dx.doi.org/10.1016/j.ridd.2013.07.005

Oppewal, A., Hilgenkamp, T. I., van Wijck, R., Schoufour, J. D., \& Evenhuis, H. M. (2014). Physical fitness is predictive for a decline in daily functioning in older adults with intellectual disabilities: Results of the HA-ID study. Res Dev Disabil, 35(10), 2299-2315. doi:http://dx.doi.org/10.1016/i.ridd.2014.05.027

Oppewal, A., Hilgenkamp, T. I., van Wijck, R., Schoufour, J. D., \& Evenhuis, H. M. (2015). Physical fitness is predictive for a decline in the ability to perform instrumental activities of daily living in older adults with intellectual disabilities: Results of the HA-ID study. Res Dev Disabil, 41-42, 7685.

Pereira, K., Basso, R. P., Lindquist, A. R., da Silva, L. G., \& Tudella, E. (2013). Infants with Down syndrome: percentage and age for acquisition of gross motor skills. Res Dev Disabil, 34(3), 894901.

Perry, J. (1992). Gait analysis: normal and pathological function. Thorofare, USA: SLACK Incorporated.

Peterson, J. J., Janz, K. F., \& Lowe, J. B. (2008). Physical activity among adults with intellectual disabilities living in community settings. Prev Med, 47(1), 101-106.

Rigoldi, C., Galli, M., Cimolin, V., Camerota, F., Celletti, C., Tenore, N., \& Albertini, G. (2012). Gait strategy in patients with Ehlers-Danlos syndrome hypermobility type and Down syndrome. Res Dev Disabil, 33(5), 1437-1442.

Rigoldi, C., Galli, M., Mainardi, L., Crivellini, M., \& Albertini, G. (2011). Postural control in children, teenagers and adults with Down syndrome. Res Dev Disabil, 32(1), 170-175.

Ringenbach, S. D., Holzapfel, S. D., Mulvey, G. M., Jimenez, A., Benson, A., \& Richter, M. (2016). The effects of assisted cycling therapy (ACT) and voluntary cycling on reaction time and measures of executive function in adolescents with Down syndrome. J Intellect Disabil Res, 60(11), 10731085.

Rintala, P., \& Loovis, E. M. (2013). Measuring motor skills in Finnish children with intellectual disabilities. Percept Mot Skills, 116(1), 294-303.

Rosano, C., Aizenstein, H., Brach, J., Longenberger, A., Studenski, S., \& Newman, A. B. (2008). Special article: gait measures indicate underlying focal gray matter atrophy in the brain of older adults. $J$ Gerontol A Biol Sci Med Sci, 63(12), 1380-1388.

Rubino, F. A. (2002). Gait disorders. Neurologist, 8(4), 254-262.

Salaun, L., \& Berthouze-Aranda, S. E. (2012). Physical fitness and fatness in adolescents with intellectual disabilities. J Appl Res Intellect Disabil, 25(3), 231-239.

Salb, J., Lindemann, U., Woodward, C., Almutaseb, S., Becker, C., Sieber, C., \& Freiberger, E. (2015). Gait Analysis in Adults with Intellectual Disabilities Living in a Residential Facility. J Appl Res Intellect Disabil. doi:10.1111/jar.12227

Slaman, J., Bussmann, J., van der Slot, W. M., Stam, H. J., Roebroeck, M. E., van den Berg-Emons, R. J., . . Lifespan Research Group South West, N. (2013). Physical strain of walking relates to activity level in adults with cerebral palsy. Arch Phys Med Rehabil, 94(5), 896-901.

Smith, B. A., Ashton-Miller, J. A., \& Ulrich, B. D. (2010). Gait adaptations in response to perturbations in adults with Down syndrome. Gait Posture, 32(2), 149-154. 
Smith, B. A., Stergiou, N., \& Ulrich, B. D. (2011). Patterns of gait variability across the lifespan in persons with and without down syndrome. J Neurol Phys Ther, 35(4), 170-177.

Smith, B. A., \& Ulrich, B. D. (2008). Early onset of stabilizing strategies for gait and obstacles: older adults with Down syndrome. Gait Posture, 28(3), 448-455.

Smits-Engelsman, B., \& Hill, E. L. (2012). The relationship between motor coordination and intelligence across the IQ range. Pediatrics, 130(4), e950-956.

Temple, V. A., Frey, G. C., \& Stanish, H. I. (2006). Physical activity of adults with mental retardation: review and research needs. Am J Health Promot, 21(1), 2-12.

Thomas, S., Reading, J., \& Shephard, R. J. (1992). Revision of the Physical Activity Readiness Questionnaire (PAR-Q). Can J Sport Sci, 17(4), 338-345.

van Schijndel-Speet, M., Evenhuis, H. M., van Wijck, R., van Montfort, K. C., \& Echteld, M. A. (2016). A structured physical activity and fitness programme for older adults with intellectual disabilities: results of a cluster-randomised clinical trial. J Intellect Disabil Res.

van Uden, C. J., \& Besser, M. P. (2004). Test-retest reliability of temporal and spatial gait characteristics measured with an instrumented walkway system (GAITRite). BMC Musculoskelet Disord, 5, 13.

VanSwearingen, J. M., \& Studenski, S. A. (2014). Aging, motor skill, and the energy cost of walking: implications for the prevention and treatment of mobility decline in older persons. J Gerontol $A$ Biol Sci Med Sci, 69(11), 1429-1436.

Verghese, J., LeValley, A., Hall, C. B., Katz, M. J., Ambrose, A. F., \& Lipton, R. B. (2006). Epidemiology of gait disorders in community-residing older adults. J Am Geriatr Soc, 54(2), 255-261.

Verghese, J., Wang, C., Lipton, R. B., Holtzer, R., \& Xue, X. (2007). Quantitative gait dysfunction and risk of cognitive decline and dementia. J Neurol Neurosurg Psychiatry, 78(9), 929-935.

Verlinden, V. J., van der Geest, J. N., Hoogendam, Y. Y., Hofman, A., Breteler, M. M., \& Ikram, M. A. (2013). Gait patterns in a community-dwelling population aged 50 years and older. Gait Posture, 37(4), 500-505.

Vismara, L., Romei, M., Galli, M., Montesano, A., Baccalaro, G., Crivellini, M., \& Grugni, G. (2007). Clinical implications of gait analysis in the rehabilitation of adult patients with "Prader-Willi" Syndrome: a cross-sectional comparative study ("Prader-Willi" Syndrome vs matched obese patients and healthy subjects). J Neuroeng Rehabil, 4, 14.

Wearing, S. C., Hennig, E. M., Byrne, N. M., Steele, J. R., \& Hills, A. P. (2006). The biomechanics of restricted movement in adult obesity. Obes Rev, 7(1), 13-24.

Westendorp, M., Houwen, S., Hartman, E., \& Visscher, C. (2011). Are gross motor skills and sports participation related in children with intellectual disabilities? Res Dev Disabil, 32(3), 1147-1153. doi:http://dx.doi.org/10.1016/j.ridd.2011.01.009

WHO. (1995). Physical status: The use and interpretation of anthropometry. WHP Technical Report Series 854. Retrieved from Geneva, Switzerland:

WHO. (1996). ICD-10 Guide for Mental Retardation. Retrieved from Geneva, Switzerland:

World Medical Association. (2013). World medical association declaration of helsinki: Ethical principles for medical research involving human subjects. JAMA, 310(20), 2191-2194. 
Table 1. Description of the gait parameters.

\begin{tabular}{|c|c|c|}
\hline Spatial parameters & Definition & Indication of worse gait ${ }^{a}$ \\
\hline Step length & $\begin{array}{l}\text { Distance between the heel centers of two } \\
\text { consecutive opposite footprints on the line } \\
\text { of progression (in } \mathrm{cm} \text { ). }\end{array}$ & lower \\
\hline Stride length & $\begin{array}{l}\text { Distance between the heel centers of two } \\
\text { consecutive footprints of the same foot on } \\
\text { the line of progression (in } \mathrm{cm} \text { ). }\end{array}$ & lower \\
\hline Base of support & $\begin{array}{l}\text { Distance from the heel center of one } \\
\text { footprint to the line of progression formed } \\
\text { by the heel centers of two opposite } \\
\text { footprints (in } \mathrm{cm} \text { ). }\end{array}$ & $\begin{array}{l}\text { either side of the optimum } \\
\text { on the parabolic curve }\end{array}$ \\
\hline Toe In/ Toe Out & $\begin{array}{l}\text { Angle between the midline of the footprint } \\
\text { and the line of progression (in degrees). } \\
\text { Positive when toe-out, and negative when } \\
\text { toe-in. }\end{array}$ & $\begin{array}{l}\text { higher (both positive and } \\
\text { negative) }\end{array}$ \\
\hline \multicolumn{3}{|l|}{ Temporal parameters } \\
\hline Velocity & $\begin{array}{l}\text { Distance traveled divided by ambulation } \\
\text { time (in } \mathrm{cm} / \mathrm{sec} \text { ). }\end{array}$ & lower \\
\hline Stride velocity & $\begin{array}{l}\text { Stride length divided by stride time (in } \\
\mathrm{cm} / \mathrm{sec} \text { ). }\end{array}$ & lower \\
\hline Cadence & Number of steps/minute & lower \\
\hline Step time & $\begin{array}{l}\text { Time elapsed between first contact of one } \\
\text { foot and first contact of the opposite foot (in } \\
\text { sec). }\end{array}$ & $\begin{array}{l}\text { either side of the optimum } \\
\text { on the parabolic curve }\end{array}$ \\
\hline Stride time (gait cycle) & $\begin{array}{l}\text { Time elapsed between the first contacts of } \\
\text { two consecutive footfalls of the same foot } \\
\text { (in sec). }\end{array}$ & $\begin{array}{l}\text { either side of the optimum } \\
\text { on the parabolic curve }\end{array}$ \\
\hline Stance time & $\begin{array}{l}\text { Time elapsed between the first contact and } \\
\text { last contact of two consecutive footfalls on } \\
\text { the same foot (in sec). It is initiated by heel } \\
\text { contact and ends with toe off of the same } \\
\text { foot. }\end{array}$ & $\begin{array}{l}\text { either side of the optimum } \\
\text { on the parabolic curve }\end{array}$ \\
\hline Swing time & $\begin{array}{l}\text { Time elapsed between the last contact of } \\
\text { the current footfall and the first contact of } \\
\text { the next footfall of the same foot (in sec). }\end{array}$ & $\begin{array}{l}\text { either side of the optimum } \\
\text { on the parabolic curve }\end{array}$ \\
\hline Single support time & $\begin{array}{l}\text { Time elapsed between the last contact of } \\
\text { the opposite foot and the first contact of the } \\
\text { next footfall of the opposite foot (in sec). }\end{array}$ & $\begin{array}{l}\text { either side of the optimum } \\
\text { on the parabolic curve }\end{array}$ \\
\hline Double support time & $\begin{array}{l}\text { Amount of time that two feet are on the } \\
\text { ground at the same time within one footfall } \\
\text { (in sec). }\end{array}$ & $\begin{array}{l}\text { either side of the optimum } \\
\text { on the parabolic curve }\end{array}$ \\
\hline \multicolumn{3}{|l|}{ Phasic parameters } \\
\hline Stance, \% GC & $\begin{array}{l}\text { Stance time as a percentage of the gait } \\
\text { cycle time (in \%). }\end{array}$ & $\begin{array}{l}\text { either side of the optimum } \\
\text { on the parabolic curve }\end{array}$ \\
\hline Swing, \%GC & $\begin{array}{l}\text { Swing time as a percentage of the gait } \\
\text { cycle time (in \%). }\end{array}$ & $\begin{array}{l}\text { either side of the optimum } \\
\text { on the parabolic curve }\end{array}$ \\
\hline Single support, \%GC & $\begin{array}{l}\text { Single support time as a percentage of the } \\
\text { gait cycle time (in \%). }\end{array}$ & $\begin{array}{l}\text { either side of the optimum } \\
\text { on the parabolic curve }\end{array}$ \\
\hline Double support, \%GC & $\begin{array}{l}\text { Double support time as a percentage of the } \\
\text { gait cycle time (in \%). }\end{array}$ & $\begin{array}{l}\text { either side of the optimum } \\
\text { on the parabolic curve }\end{array}$ \\
\hline \multicolumn{3}{|l|}{ Variability parameters } \\
\hline Step length SD & Standard deviation in step length (in $\mathrm{cm}$ ) & higher \\
\hline Stride length SD & Standard deviation in stride length (in cm) & higher \\
\hline Base of support SD & $\begin{array}{l}\text { Standard deviation in base of support (in } \\
\mathrm{cm} \text { ) }\end{array}$ & higher \\
\hline
\end{tabular}


Standard deviation in stride time (in sec)

Standard deviation in single support time (in higher

Standard deviation in single support time (in higher $\mathrm{sec}$ )

$S D=$ Standard deviation; \%GC = percentage of the gait cycle.

a = Indications for worse gait. 'Lower' means that lower values are considered worse gait, and 'higher' means that higher values are considered worse gait.
Stride time SD

Stride velocity SD

Step time SD

Stance time SD

Swing time SD

Single support time SD Standard deviation in stride velocity (in $\mathrm{cm} / \mathrm{s}$ )

Standard deviation in step time (in sec) Standard deviation in stance time (in sec) Standard deviation in swing time (in sec) $\mathrm{sec})$

higher

higher

higher

higher

higher

Double support time SD 
Table 2. Personal characteristics and medical information of the study sample.

Total study sample

$(N=31)$

\begin{tabular}{|c|c|c|}
\hline \multicolumn{3}{|l|}{ Personal characteristics } \\
\hline Age & Years, $m \pm s d$, range & $42.77 \pm 16.70,20-68$ \\
\hline \multirow[t]{2}{*}{ Sex } & Female, $n(\%)$ & $7(22.6 \%)$ \\
\hline & Male, $n(\%)$ & $24(77.4 \%)$ \\
\hline \multirow[t]{2}{*}{ Level of ID } & Mild, $n(\%)$ & $15(48.4 \%)$ \\
\hline & Moderate, $n(\%)$ & $16(51.6 \%)$ \\
\hline Height & $\mathrm{cm}, m \pm s d$ & $170.18 \pm 9.22$ \\
\hline Weight & $\mathrm{kg}, m \pm s d$ & $78.97 \pm 14.81$ \\
\hline \multirow[t]{4}{*}{ BMI } & $\mathrm{kg} / \mathrm{m}^{2}, m \pm s d$ & $27.24 \pm 4.51$ \\
\hline & Normal, $n(\%)$ & $9(29.0 \%)$ \\
\hline & Overweight, $n$ (\%) & $15(48.4 \%)$ \\
\hline & Obese, $n(\%)$ & $7(22.6 \%)$ \\
\hline \multicolumn{3}{|l|}{ Medical information } \\
\hline \multirow[t]{7}{*}{ Genetic syndrome } & No genetic syndrome, $n(\%)$ & $9(29.0 \%)$ \\
\hline & PKÜ, $n(\%)$ & $1(3.2 \%)$ \\
\hline & $\begin{array}{l}\text { Mosaic mutation XLIS gene, } n \\
(\%)\end{array}$ & $1(3.2 \%)$ \\
\hline & Smith-Magenis syndrome, $n(\%)$ & $1(3.2 \%)$ \\
\hline & Williams syndrome, $n(\%)$ & $1(3.2 \%)$ \\
\hline & Perlman syndrome, $n(\%)$ & $1(3.2 \%)$ \\
\hline & Unknown, $n(\%)$ & $17(54.8 \%)$ \\
\hline Osteoarthritis & Yes, $n(\%)$ & $4(12.9 \%)$ \\
\hline Visual impairments ${ }^{a}$ & Yes, $n(\%)$ & $4(12.9 \%)$ \\
\hline Spasticity arms & Yes, $n(\%)$ & 0 \\
\hline Spasticity legs & Yes, $n(\%)$ & $1(3.2 \%)$ \\
\hline Orthopedic shoes & Yes, $n(\%)$ & $6(19.4 \%)$ \\
\hline \multicolumn{3}{|l|}{ Medication use } \\
\hline \multirow[t]{2}{*}{ Antidepressants } & Yes, $n(\%)$ & $6(19.4 \%)$ \\
\hline & No, $n(\%)$ & $25(80.6 \%)$ \\
\hline \multirow[t]{2}{*}{ Antipsychotics } & Yes, $n(\%)$ & $15(48.4 \%)$ \\
\hline & No, $n(\%)$ & $16(51.6 \%)$ \\
\hline \multirow[t]{2}{*}{ Antiepileptics } & Yes, $n(\%)$ & $3(9.7 \%)$ \\
\hline & No, $n(\%)$ & $28(90.3 \%)$ \\
\hline \multirow[t]{2}{*}{ Benzodiazepines } & Yes, $n(\%)$ & $6(19.4 \%)$ \\
\hline & No, $n(\%)$ & $25(80.6 \%)$ \\
\hline \multirow[t]{2}{*}{ Polypharmacy ( $\geq 5$ medications) } & Yes, $n(\%)$ & $13(41.9 \%)$ \\
\hline & No, $n(\%)$ & $18(58.1 \%)$ \\
\hline
\end{tabular}

$\mathrm{n}=$ number of participants; $m$ = mean; $s d=$ standard deviation; ID = intellectual disability;

SPPB $=$ Short Physical Performance Battery.

$\mathrm{a}=$ participants with a visual impairment but still with a vision $>0.3$. 
Table 3. Results of the gait parameters while walking at comfortable speed.

\begin{tabular}{lll}
\hline & M (SD) & $\mathbf{9 5 \% ~ C l}$ \\
\hline Spatial parameters & & \\
Step length (cm) & $65.28 \pm 10.14$ & {$[61.56,69.0]$} \\
Stride length (cm) & $130.88 \pm 20.25$ & {$[123.45,138.31]$} \\
Base of support (cm) & $11.88 \pm 3.51$ & {$[10.59,13.17]$} \\
Toe in/ Toe out (degrees) & $7.06 \pm 7.17$ & {$[4.43,9.69]$} \\
Temporal parameters & & \\
Velocity (cm/sec) & $118.36 \pm 23.43$ & {$[109.76,126.95]$} \\
Stride velocity (cm/sec) & $118.98 \pm 23.47$ & {$[110.37,127.59]$} \\
Cadence (steps/min) & $108.36 \pm 10.19$ & {$[104.62,112.10]$} \\
Step time (sec) & $0.56 \pm 0.05$ & {$[0.54,0.58]$} \\
Stride (cycle) time (sec) & $1.12 \pm 0.11$ & {$[1.08,1.15]$} \\
Stance time (sec) & $0.66 \pm 0.08$ & {$[0.63,0.69]$} \\
Swing time (sec) & $0.46 \pm 0.04$ & {$[0.44,0.47]$} \\
Single support time (sec) & $0.46 \pm 0.04$ & {$[0.44,0.47]$} \\
Double support time (sec) & $0.20 \pm 0.06$ & {$[0.18,0.22]$} \\
Phasic parameters & & \\
Stance, \%GC & $58.97 \pm 1.99$ & {$[58.24,59.70]$} \\
Swing, \%GC & $41.03 \pm 1.99$ & {$[40.30,41.76]$} \\
Single support, \%GC & $41.03 \pm 1.99$ & {$[40.30,41.76]$} \\
Double support, \%GC & $18.08 \pm 4.08$ & {$[16.58,19.57]$} \\
Variability parameters & & \\
Step length SD & $2.99 \pm 0.89$ & {$[2.66,3.32]$} \\
Stride length SD & $5.29 \pm 1.90$ & {$[4.59,5.99]$} \\
Base of support SD & $2.51 \pm 1.07$ & {$[2.12,2.91]$} \\
Stride velocity SD & $7.07 \pm 2.84$ & {$[6.03,8.11]$} \\
Step time SD & $0.02 \pm 0.01$ & {$[0.02,0.03]$} \\
Stride time SD & $0.04 \pm 0.02$ & {$[0.03,0.04]$} \\
Stance time SD & $0.03 \pm 0.01$ & {$[0.026,0.034]$} \\
Swing time SD & $0.02 \pm 0.01$ & {$[0.02,0.03]$} \\
Single support time SD & $0.02 \pm 0.01$ & {$[0.02,0.03]$} \\
Double support time SD & $0.03 \pm 0.02$ & {$[0.02,0.03]$} \\
\hline$m=$ mean; sd = standard deviation; CI confidence interval; \% GC = percentage of the gait cycle.
\end{tabular}


Table 4. Effect sizes of the comparisons between age, sex, and level of ID with the gait parameters.

\begin{tabular}{|c|c|c|c|c|c|c|c|c|}
\hline & $\begin{array}{l}\text { Sex }^{c} \\
\text { (d) }\end{array}$ & $\begin{array}{l}\text { Effect } \\
\text { category }^{a}\end{array}$ & $\begin{array}{l}\text { Level of ID } \\
\text { (d) }\end{array}$ & $\begin{array}{l}\text { Effect } \\
\text { category }^{a}\end{array}$ & $\begin{array}{l}\text { Age } \\
(r)\end{array}$ & $\begin{array}{l}\text { Effect } \\
\text { category }^{b}\end{array}$ & $\begin{array}{l}\text { BMI } \\
(r)\end{array}$ & $\begin{array}{l}\text { Effect } \\
\text { category }^{b}\end{array}$ \\
\hline \multicolumn{9}{|l|}{ Spatial parameters } \\
\hline Step length (cm) & 0.60 & medium & 0.50 & medium & -0.27 & small & -0.11 & small \\
\hline Stride length (cm) & 0.59 & medium & 0.49 & small & -0.27 & small & -0.12 & small \\
\hline Base of support (cm) & -0.29 & small & -0.19 & small & 0.28 & small & 0.20 & small \\
\hline Toe in/ Toe out (degrees) & 0.44 & small & -0.02 & small & 0.15 & small & -0.14 & small \\
\hline \multicolumn{9}{|l|}{ Temporal parameters } \\
\hline Velocity $(\mathrm{cm} / \mathrm{sec})$ & 0.45 & small & 0.31 & small & -0.22 & small & -0.19 & small \\
\hline Stride velocity $(\mathrm{cm} / \mathrm{sec})$ & 0.44 & small & 0.30 & small & -0.22 & small & -0.19 & small \\
\hline Cadence (steps/min) & -0.61 & medium & -0.14 & small & -0.12 & small & -0.14 & small \\
\hline Step time (sec) & -0.68 & medium & 0 & small & 0.03 & small & 0.27 & small \\
\hline Stride (cycle) time (sec) & -0.62 & medium & 0 & small & 0.03 & small & 0.27 & small \\
\hline Stance time (sec) & -0.74 & medium & 0.10 & small & 0.08 & small & $0.42^{\star}$ & medium \\
\hline Swing time (sec) & -0.50 & medium & -0.16 & small & -0.06 & small & -0.01 & small \\
\hline Single support time (sec) & -0.50 & medium & -0.16 & small & -0.06 & small & -0.01 & small \\
\hline Double support time (sec) & -0.81 & large & 0.31 & small & 0.19 & small & $0.67^{\star \star}$ & large \\
\hline \multicolumn{9}{|l|}{ Phasic parameters } \\
\hline Stance, \%GC & -1.40 & $\operatorname{large}^{*}$ & 0.05 & small & 0 & small & $0.42^{\star}$ & medium \\
\hline Swing, \%GC & -0.53 & medium & -0.27 & small & -0.19 & small & -0.28 & small \\
\hline Single support, \%GC & -0.52 & medium & -0.28 & small & -0.18 & small & -0.28 & small \\
\hline Double support, \%GC & -0.98 & $\operatorname{large}^{\star}$ & 0.40 & small & 0.19 & small & $0.73^{\star *}$ & large \\
\hline \multicolumn{9}{|l|}{ Variability parameters } \\
\hline Step length SD & -0.31 & small & -0.18 & small & 0.17 & small & 0.02 & small \\
\hline Stride length SD & -0.25 & small & -0.04 & small & 0.12 & small & 0.08 & small \\
\hline Base of support SD & -0.04 & small & -0.59 & medium & 0.21 & small & -0.07 & small \\
\hline Stride velocity SD & 0.44 & small & 0.02 & small & -0.08 & small & 0.05 & small \\
\hline Step time SD & -2.04 & large $e^{\star \star}$ & 0 & small & 0.06 & small & 0.28 & small \\
\hline Stride time SD & -1.18 & large* $^{*}$ & 0 & small & 0.08 & small & 0.21 & small \\
\hline Stance time SD & -0.79 & large & 0 & small & 0.23 & small & 0.24 & small \\
\hline Swing time SD & -1.04 & $\operatorname{large}^{*}$ & 0 & small & 0.23 & small & 0.17 & small \\
\hline Single support time SD & -1.04 & large $^{*}$ & 0 & small & 0.23 & small & 0.17 & small \\
\hline Double support time SD & -0.60 & medium & 0 & small & 0.13 & small & $0.45^{\star}$ & medium \\
\hline
\end{tabular}

ID = intellectual disability; $d=$ Cohen's $d$ as effect size; $r=$ Pearson's correlation coefficient as effect size.

a small (0.2), medium (0.5), large (0.8) effect; b small (0.1), medium (0.3), large (0.5) effect.

c positive effect size means that males have a higher mean value of the specific parameter than females.

d positive effect size means that adults mild ID have a higher mean value of the specific parameter than adults with moderate ID.

* $p<0.05 ;{ }^{* *} p<0.002$ 
